\title{
La science au service de la conservation au parc marin du Saguenay-Saint-Laurent : Comment mieux comprendre permet de mieux protéger
}

\author{
Science Serving Conservation in the Saguenay-St. Lawrence \\ Marine Park: How does better knowledge lead to better \\ protection?
}

\section{Nadia Ménard}

Volume 22, numéro 2, 2009

URI : https://id.erudit.org/iderudit/037477ar

DOI : https://doi.org/10.7202/037477ar

\section{Aller au sommaire du numéro}

\section{Éditeur(s)}

Université du Québec - INRS-Eau, Terre et Environnement (INRS-ETE)

\section{ISSN}

1718-8598 (numérique)

\section{Découvrir la revue}

Citer cet article

Ménard, N. (2009). La science au service de la conservation au parc marin du Saguenay-Saint-Laurent : Comment mieux comprendre permet de mieux protéger. Revue des sciences de l'eau / Journal of Water Science, 22(2), 115-123. https://doi.org/10.7202/037477ar

\section{Résumé de l'article}

Résultant d'une vingtaine d'années d'études, de support du milieu régional et de négociations, la création du parc marin en 1998 peut être considérée comme un événement important dans le domaine de la conservation du milieu marin, au Québec comme au Canada. Située à la confluence de l'estuaire du Saint-Laurent et du Saguenay, la région couverte par le parc marin est reconnue comme étant un lieu particulier à plusieurs égards. Les écosystèmes estuariens qu'on y retrouve sont d'une grande richesse, mais sont également soumis à plusieurs sources de pression.

L'atteinte des objectifs énoncés lors de la création du parc marin exige le recours à une gamme d'outils comme la gouvernance, la science et l'éducation. La recherche scientifique est inscrite au sein même du mandat légal du parc marin, ce qui témoigne de son importance comme outil de conservation. Mais pour que la science puisse rendre service à la conservation, il est nécessaire de diffuser les connaissances au public. Par ailleurs, puisque la science ne détient pas toujours de certitude devant la complexité des écosystèmes, il importe parfois de recourir à la gestion adaptative et au principe de précaution. Le rapprochement entre les divers joueurs, comme les scientifiques, les gestionnaires, les communautés et les visiteurs, est essentiel afin que les connaissances scientifiques puissent orienter les actions de conservation, la gestion des activités et susciter l'adhésion aux objectifs du parc marin. Beaucoup de chemin a été parcouru depuis l'établissement du parc marin il y a dix ans, mais l'intégration des connaissances scientifiques aux actions de conservation de ce milieu exceptionnel que constitue le parc marin demeure toujours un important défi. 


\title{
LA SCIENCE AU SERVICE DE LA CONSERVATION AU PARC MARIN DU SAGUENAY-SAINT-LAURENT : COMMENT MIEUX COMPRENDRE PERMET DE MIEUX PROTÉGER *
}

\author{
Science Serving Conservation in the Saguenay-St. Lawrence Marine Park: \\ How does better knowledge lead to better protection?
}

\author{
NADIA MÉNARD ${ }^{1 *}$ \\ ${ }^{1}$ Nadia Ménard, Parcs Canada, Parc marin du Saguenay-Saint-Laurent \\ 182, rue de l'Église, C. P. 220, Tadoussac (Québec) G0T 2A0
}

Reçu le 24 juillet 2008, accepté le 4 décembre 2008

\section{RÉSUMÉ}

Résultant d'une vingtaine d'années d'études, de support du milieu régional et de négociations, la création du parc marin en 1998 peut être considérée comme un événement important dans le domaine de la conservation du milieu marin, au Québec comme au Canada. Située à la confluence de l'estuaire du Saint-Laurent et du Saguenay, la région couverte par le parc marin est reconnue comme étant un lieu particulier à plusieurs égards. Les écosystèmes estuariens qu'on y retrouve sont d'une grande richesse, mais sont également soumis à plusieurs sources de pression.

L'atteinte des objectifs énoncés lors de la création du parc marin exige le recours à une gamme d'outils comme la gouvernance, la science et l'éducation. La recherche scientifique est inscrite au sein même du mandat légal du parc marin, ce qui témoigne de son importance comme outil de conservation. Mais pour que la science puisse rendre service à la conservation, il est nécessaire de diffuser les connaissances au public. Par ailleurs, puisque la science ne détient pas toujours de certitude devant la complexité des écosystèmes, il importe parfois de recourir à la gestion adaptative et au principe de précaution. Le rapprochement entre les divers joueurs, comme les scientifiques, les gestionnaires, les communautés et les visiteurs, est essentiel afin que les connaissances scientifiques puissent orienter les actions de conservation, la gestion des activités et susciter l'adhésion aux objectifs du parc marin. Beaucoup de chemin a été parcouru depuis l'établissement du parc marin il y a dix ans, mais l'intégration des connaissances scientifiques aux actions de conservation de ce milieu exceptionnel que constitue le parc marin demeure toujours un important défi.

Mots clés : Parc marin du Saguenay-Saint-Laurent, aire marine protégée, conservation, science, appui du public, gestion.

* Parc marin Saguenay-Saint-Laurent

*Auteure pour correspondance :

Téléphone: $418235-4703$, poste 244

Courriel: nadia.menard@pc.gc.ca 


\section{ABSTRACT}

The establishment of the Saguenay-St. Lawrence Marine Park in 1998 is the result of twenty years of studies, public support and negotiations. Its establishment is a significant event in the field of marine conservation in Quebec and in Canada. Located at the confluence of the St. Lawrence Estuary and the Saguenay Fjord, the area covered by the Marine Park is recognized for many reasons. The estuarine ecosystems found here are very rich from a biological point but are also under many forms of pressure.

Attaining the objectives sought out by the establishment of the Marine Park requires to use a wide variety of tools such as governance, science and education. Scientific research is included in the legal mandate of the Marine Park, indicating its importance as a conservation tool. But using science as a conservation tool requires communicating results to the public. Furthermore, since science does not always provide certainty relative to the complexity of marine ecosystems, it is sometimes necessary to have recourse to adaptive management and to the precautionary principle. Bringing together various players such as scientists, managers, local communities and visitors is essential to enable scientific knowledge to orient conservation actions. The Marine Park has come a long way since its establishment ten years ago, but it remains a challenge to ensure that scientific knowledge is used to take action to conserve the exceptional environment of the Marine Park.

\section{Keywords: Saguenay-St. Lawrence Marine Park, marine protec- ted areas, conservation, science, public support, management.}

\section{INTRODUCTION}

Depuis sa création en 1998, le parc marin du Saguenay-Saint-Laurent confère un statut d'aire protégée à une portion d'écosystèmes dits représentatifs de l'estuaire du Saint-Laurent et du fjord du Saguenay. La richesse et la complexité des écosystèmes du parc marin, ainsi que la fragilité des composantes naturelles face aux activités anthropiques, ont attiré l'attention de nombreux chercheurs, du public et des décideurs. L'accès aux connaissances sur les conditions océanographiques, sur l'unicité du fjord et sur la précarité de la population de bélugas a été déterminant dans la création du parc. Létablissement du parc marin, une des premières aires marines protégées au Canada, est une reconnaissance

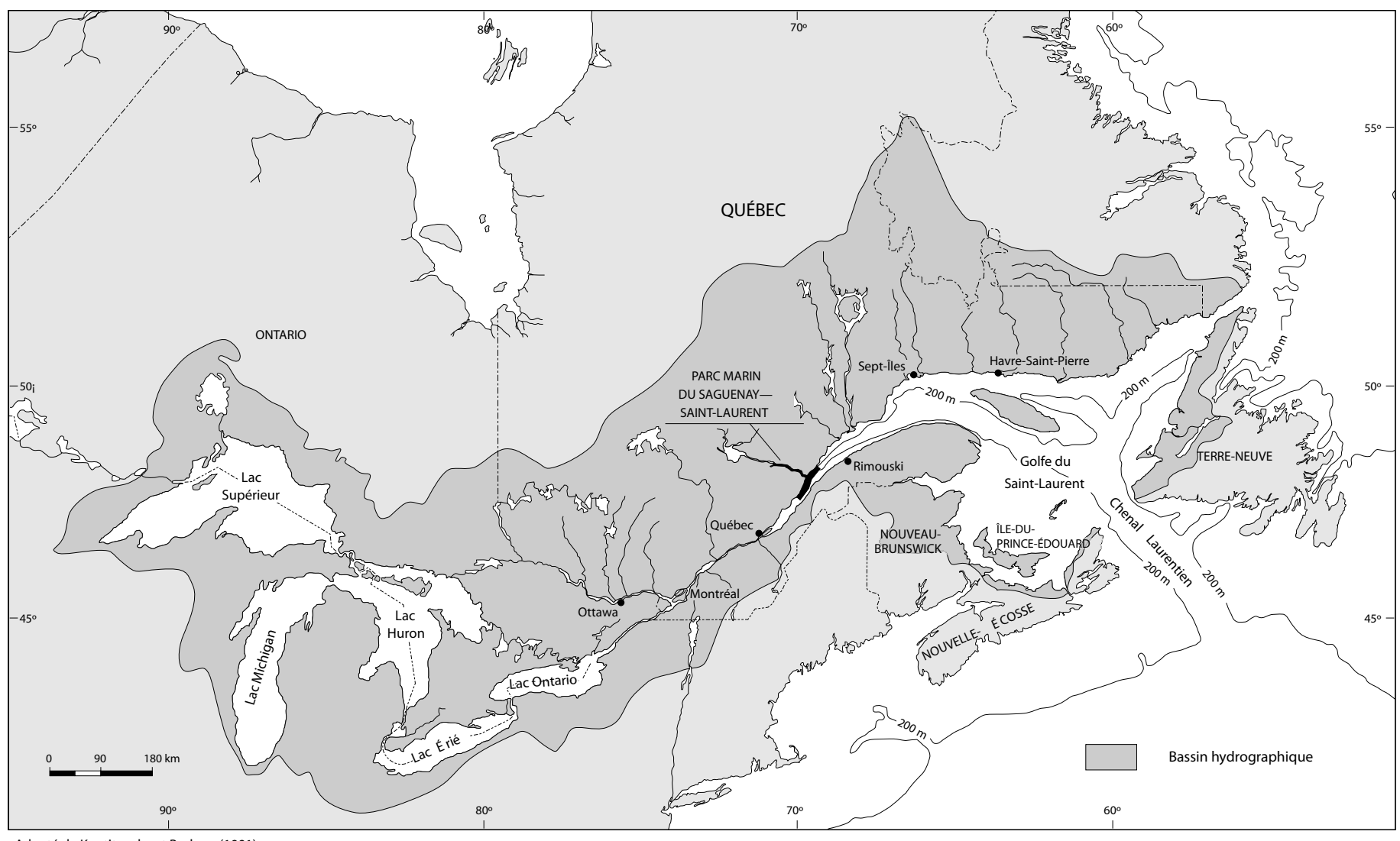

Adapté de Koutitonsky et Budgen (1991).

Figure 1. Situation géographique du parc marin du Saguenay-Saint-Laurent dans le bassin versant du Saint-Laurent et des Grands Lacs (source : DIONNE, 2001).

General location of the Saguenay-St. Lawrence Marine Park in the drainage basin of the St. Lawrence River and the Canadian Great Lakes (from DIONNE, 2001) 
de la valeur exceptionnelle de son territoire. Résultant d'une vingtaine d'années d'études, de support du milieu régional et de négociations, la création du parc marin peut être considérée comme un événement important dans le domaine de la conservation du milieu marin, au Québec comme au Canada.

Le numéro spécial de la Revue des Sciences de l'eau et le colloque scientifique "Dix années de science au service de la conservation" offrent tous deux l'opportunité de faire le bilan des recherches réalisées au cours des dix années d'existence du parc marin. Ce bilan servira à déterminer les besoins futurs de recherche, mais aussi à informer le public et les décideurs afin d'orienter les actions pour atteindre les objectifs de conservation du parc marin.

\section{TERRITOIRE DU PARC MARIN}

Le parc marin se situe environ à mi-chemin entre les Grands Lacs et l'océan Atlantique et est sous l'influence de ces deux systèmes (Figure 1). La confluence de l'estuaire du Saint-Laurent et du Saguenay est reconnue comme étant un lieu particulier à plusieurs égards. Notamment, les eaux drainant l'immense bassin versant des Grands Lacs et du Saint-Laurent et celui du Saguenay-Lac-Saint-Jean, ainsi que celles en provenance de l'océan Atlantique, y convergent, générant des conditions océanographiques qui se démarquent dans le Saint-Laurent.

Couvrant une superficie de $1246 \mathrm{~km}^{2}$, le parc marin du Saguenay-Saint-Laurent comprend toute la colonne d'eau et les fonds marins jusqu’à la ligne des hautes marées ordinaires (Figure 2). Son territoire est entièrement constitué d'écosystèmes estuariens, soit l'estuaire moyen, l'estuaire maritime et le fjord du Saguenay, qui sont tous trois des milieux extrêmement dynamiques. Les caractéristiques physiques et biologiques different beaucoup entre ces trois portions du parc marin, malgré leur interdépendance (PMSSL, 2008). Par exemple, de récentes études sur les populations de poissons mettent en évidence la connectivité entre les systèmes du Saguenay et de l'estuaire du Saint-Laurent (SÉVIGNY et al., 2009; SIROIS et al., 2009).

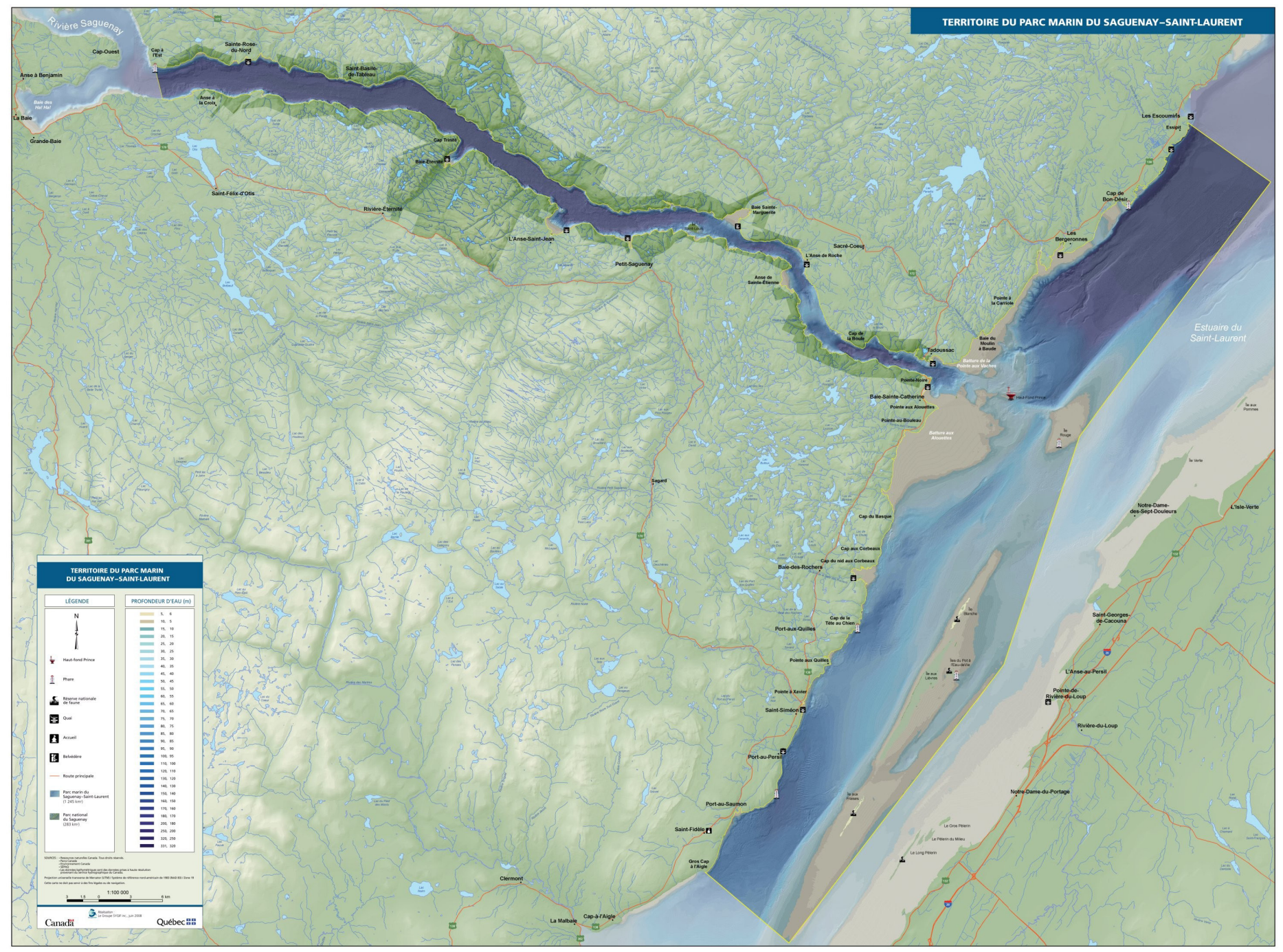

Figure 2. Territoire du parc marin du Saguenay-Saint-Laurent (source : Parcs Canada). Territory of the Saguenay-St. Lawrence Marine Park (from Parcs Canada) 


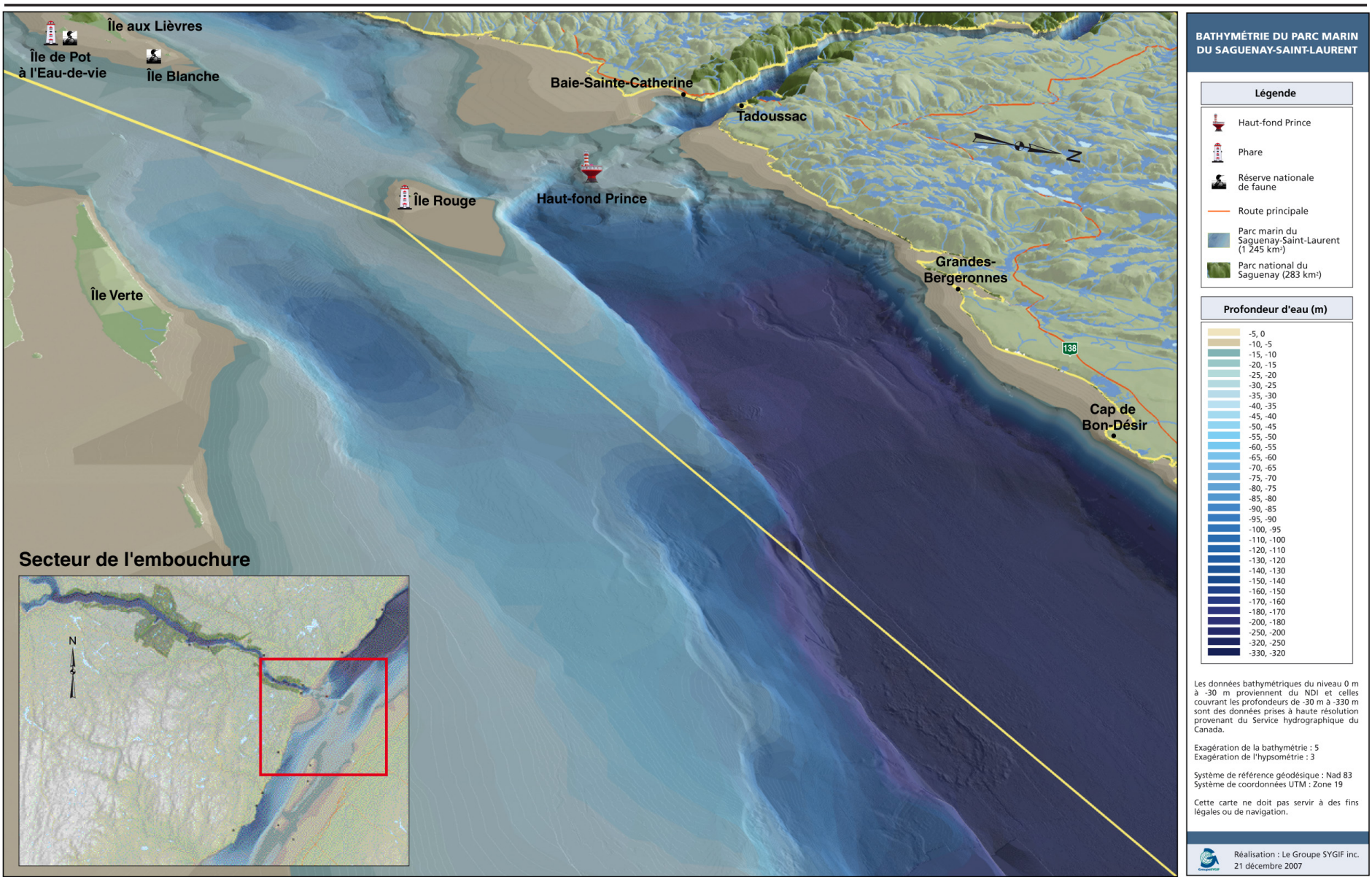

Figure 3. La tête du chenal Laurentien à la confluence du fjord du Saguenay et de l'estuaire du Saint-Laurent (source : Parcs Canada). The head of the Laurentian Channel at the confluence of the Saguenay Fjord and the St. Lawrence Estuary (from Parcs Canada).

La tête du chenal Laurentien est la particularité topographique la plus saillante du parc marin, marquant la transition entre l'estuaire moyen et l'estuaire maritime, à l'endroit même de la confluence du Saguenay et du Saint-Laurent (Figure 3). La topographie sous-marine accidentée, la présence du ford du Saguenay et la remontée d'eau froide au rythme des marées, communément appelée " upwelling ", en font une région très singulière (SAUCIER et al., 2009). À l'échelle mondiale, les sites de remontée d'eau froide sont parmi les plus productifs des océans. L' " upwelling " entraîne vers la surface des éléments nutritifs et du zooplancton, tout en favorisant l'oxygénation de l'eau. L' "upwelling" à la tête du chenal Laurentien est le processus océanographique qui distingue le plus le parc marin des autres régions de l'estuaire du Saint-Laurent. L'effet de la remontée des eaux froides se répercute sur l'ensemble de l'estuaire du Saint-Laurent et du Saguenay, même jusque dans le golfe du Saint-Laurent. Les remontées d'eau froide associées au rythme des marées représentent donc en quelque sorte le cœur battant et les poumons du parc marin.

Compte tenu de la petite taille du territoire du parc marin relativement à l'estuaire du Saint-Laurent, la diversité des espèces connues qui s'y retrouvent est remarquable (ARGUS, 1992 et références incluses; BOSSÉ et al., 1994). En effet, les conditions océanographiques à la confluence du Saint-Laurent et du Saguenay favorisent non seulement la production primaire (INGRAM, 1975; THERRIAULT et al., 1990), mais également la concentration d'espèces planctoniques situées à la base du réseau alimentaire (SIMARD et al., 2009). À son tour, la concentration de zooplancton favorise la convergence de prédateurs comme les poissons, qui servent également de proies, les oiseaux de mer et les mammifères marins. Essentiellement, les principaux facteurs expliquant la grande diversité d'espèces retrouvées dans le parc marin sont: la diversité des écosystèmes et des habitats, l'abondance d'espèces-proies, le chevauchement des aires de distribution d'espèces marines et d'espèces d'eau douce, et la présence d'espèces d'affinité arctique (PMSSL, 2008).

Cependant, les conditions océanographiques et bathymétriques particulières ne favorisent pas seulement la richesse biologique du parc marin, elles favorisent également l'apport et la rétention de sédiments contaminés. La tête $\mathrm{du}$ chenal Laurentien est une zone de sédimentation et d'accumulation de polluants persistants provenant du bassin versant des Grands Lacs et du Saint-Laurent (LEBEUF et NUNES, 2005). Dans le Saguenay, les substances persistantes s'évacuent très peu en raison de la géomorphologie du fjord (PELLETIER et al., 2009). La coïncidence géographique d'un écosystème d'une grande richesse avec un site d'accumulation de contaminants pouvant provenir de centaines de kilomètres 
en amont illustre l'ampleur des défis pour la conservation de ce milieu exceptionnel.

\section{HISTORIQUE DE LA CRÉATION DU PARC MARIN}

Le parc marin du Saguenay-Saint-Laurent est un projet innovateur à plusieurs égards. D'abord, il a été créé à une époque où il n'existait pas de moyens légaux pour désigner une aire marine protégée de ce genre au Canada. Ainsi, la création du parc marin a nécessité l'adoption de deux lois constituantes : une du gouvernement du Canada et une du gouvernement $\mathrm{du}$ Québec. Le parc marin est unique en ce sens qu'il est cogéré par les deux paliers de gouvernements. De plus, il vise à favoriser la gestion participative avec le milieu. Dix ans après la création légale du parc marin, un retour sur son historique aide à mieux comprendre où nous en sommes aujourd'hui.

Le Canada bénéficie de plus d'un siècle d'expérience en matière d'aires protégées terrestres, le premier parc national ayant été établi à Banff en 1885. En contrepartie, il compte seulement dix ans d'expérience dans l'établissement et la gestion des aires marines protégées. Le concept d'aire marine protégée est en effet relativement nouveau au Canada. Dans le contexte canadien, ce type de désignation est particulièrement pertinent, notre pays étant entouré de trois océans et d'une immense réserve d'eau douce que constituent les Grands Lacs.

Pendant des siècles, le Saint-Laurent a été exploité intensivement et il en subit encore les conséquences. Ce n'est que depuis une trentaine d'années que des mesures de protection et de restauration du Saint-Laurent et de ses affluents ont été entreprises. Dans les années 1980, la situation inquiétante du béluga est venue symboliser la détérioration de l'environnement du Saint-Laurent. Le béluga a suscitél'urgence d'agir pour protéger le fleuve et ses affluents. Il est à l'origine de nombreuses initiatives, dont le Plan d'action Saint-Laurent et la mobilisation populaire qui a mené à la création du parc marin du Saguenay-Saint-Laurent (PMSSL, 2008; PRESCOTT et GAUQUELIN, 1990).

Cette époque d'éveil de la population à la fragilité de l'environnement a favorisé la diffusion des connaissances sur l'état du Saint-Laurent, le développement des accès au fleuve et la renaissance d'une industrie touristique axée sur l'observation de la nature. C'était le début d'une ère de retour au fleuve. Depuis, l'attachement des québécois pour le Saint-Laurent a continué de se renforcer, probablement en raison du développement des accès au fleuve.

La volonté d'établir un parc marin ouvrait la voie à une nouvelle forme d'utilisation, ou plus précisément, une nouvelle vocation du territoire, visant à favoriser la conservation des écosystèmes, l'utilisation écologiquement durable des res sources, le maintien de la biodiversité, l'appréciation par le public, la sensibilisation et l'acquisition de connaissances. Autrement dit, une aire marine protégée représente bien plus qu'un laboratoire naturel de recherche et d'enseignement, c'est un lieu pour expérimenter de nouvelles façons d'utiliser le milieu marin. C'est précisément le type d'expérience que connaît la région avec l'établissement du parc marin et dont plusieurs projets d'aires marines protégées au Canada s'inspirent.

\section{RÔLE DE LA SCIENCE AU PARC MARIN}

Dans le cadre de la création et de la gestion des aires marines protégées, la recherche scientifique est essentielle à l'identification des territoires à protéger, à leur planification, au développement des programmes éducatifs et à la résolution d'enjeux de gestion. Dans le contexte spécifique du parc marin, la recherche scientifique est inscrite au sein même du mandat du parc, ce qui témoigne de l'importance de la science pour l'atteinte de ses objectifs :

[...] rehausser, au profit des générations actuelles et futures, le niveau de protection des écosystèmes d'une partie représentative du fjord du Saguenay et de l'estuaire du Saint-Laurent aux fins de conservation, tout en favorisant son utilisation à des fins éducatives, récréatives et scientifiques.

La recherche scientifique a été déterminante dans l'établissement du parc marin. Pendant quatre décennies, elle a permis de mieux comprendre les particularités du territoire. En effet, l'intérêt des chercheurs pour les conditions océanographiques et les mammifères marins de la région remonte aux années 1960 . Les travaux de chercheurs comme DRAINVILLE (1968), EDDS et MACFARLANE (1987), FORRESTER (1974), INGRAM (1975), LYNAS et SYLVESTRE (1988), PIPPARD et MALCOLM (1978), REID (1977), pour nommer quelques pionniers, ont permis de décrire les phénomènes océanographiques et biologiques qui favorisent la grande richesse des écosystèmes. La présence récurrente des cétacés indique d'ailleurs l'importance des conditions particulières retrouvées en ce lieu.

Dans les années 1970, les premières études sur le potentiel d'établissement d'une aire marine protégée dans le Saint-Laurent ont été réalisées. Une analyse océanographique et biologique de l'estuaire et du golfe du Saint-Laurent a été produite par des chercheurs affiliés à l'Université McGill pour le compte du Service des parcs du Canada (aujourd'hui Parcs 
Canada) afin de déterminer les sites d'intérêt pour la création d'une aire marine protégée (DUNBAR et al., 1977). De plus, le premier rapport traitant de la distribution de la population de bélugas et de ses habitats a été préparé (PIPPARD et MALCOLM, 1978) à la suite d'un contrat de Parcs Canada. D'ailleurs, les travaux de PIPPARD (1985) ont été déterminants pour l'octroi d'un statut de population en péril aux bélugas du Saint-Laurent et pour le choix de la confluence du Saguenay et du Saint-Laurent comme endroit où établir une aire marine protégée.

À partir des années 1980, le projet de création de cette aire marine protégée a stimulé la recherche dans divers domaines. En effet, la création et la gestion d'une aire marine protégée exigent l'intégration de connaissances multidisciplinaires sur les écosystèmes, l'impact des activités anthropiques, et les contextes culturel, social et économique. Depuis les années 1990, l'effort de recherche a connu un élan dans plusieurs champs d'études pour appuyer la gestion du parc marin. En somme, de 1990 à 2008, une centaine de projets ont été réalisés dans les domaines des sciences naturelles et des activités humaines, souvent en collaboration avec le milieu universitaire, d'autres ministères ou d'organismes non gouvernementaux. Plusieurs projets pluriannuels ont permis de faire des suivis s'échelonnant sur une quinzaine d'années, comme le suivi de la pêche hivernale sur glace (LAMBERT, 2002) et le suivi des activités d'observation en mer (MICHAUD et al., 2007). Ces suivis ont été déterminants dans l'établissement de mesures de gestion, comme les quotas pour la pêche hivernale dans le ford du Saguenay et l'adoption du Règlement sur les activités en mer dans le parc marin du Saguenay-Saint-Laurent.

Les projets de recherche réalisés dans la région du parc marin sont un reflet de l'intérêt porté à la diversité des composantes des écosystèmes et des activités qui s'y déroulent. En effet, ces travaux ont porté sur des sujets aussi variés que : la géomorphologie marine; l'archéologie; l'histoire; l'océano graphie physique et biologique; les espèces marines, dont le béluga du Saint-Laurent; la pollution chimique et sonore; le trafic maritime et l'impact économique et écologique de diverses activités humaines, notamment le tourisme et les pêches. De nombreux rapports faisant le bilan des connaissances sur le fjord du Saguenay et l'estuaire du Saint-Laurent ont été produits au cours des dernières années (ex. : ARGUS, 1992; DIONNE, 2001; MPO, 2007; PMSSL, 2008; SAVARIA et al., 2003; SÉVIGNY et COUILLARD, 1994). Afin de diffuser les résultats de ces recherches au public, plusieurs informations scientifiques ont été incorporées dans des produits éducatifs à l'intérieur des expositions et des activités d'interprétation offertes dans l'aire de coordination du parc marin.

\section{FAVORISER LA CONSERVATION PAR LA SCIENCE}

Dans une région aux paysages naturels remarquables, où l'on peut observer une grande abondance d'espèces sauvages à la surface de l'eau, il peut s'avérer très difficile de saisir l'impact environnemental des activités humaines qui ont lieu sur le territoire. Il est encore plus difficile de percevoir les conséquences des activités qui s'effectuent à des centaines de kilomètres du parc marin. Conséquemment, l'observation et l'analyse scientifique sont parfois le seul moyen de prendre la mesure de l'état réel du milieu marin et des processus environnementaux complexes qui s'opèrent dans le système.

L'acquisition de connaissances scientifiques est une première étape dans les retombées que celles-ci peuvent apporter à la conservation. Le rapprochement entre les divers joueurs comme les scientifiques, les gestionnaires, les communautés et les visiteurs est essentiel pour que les connaissances scientifiques puissent effectivement orienter les actions de conservation. Puisque le succès d'une aire marine protégée comme le parc marin nécessite l'appui de tous les acteurs concernés par celle-ci, la transmission des connaissances est une condition indispensable pour que la science soit un outil efficace de conservation.

L'expérience vécue au parc marin, de mêmequ'ailleurs dansle Saint-Laurent (ex. instauration du Plan d'action Saint-Laurent il y a 20 ans) et dans le monde (ex : BEARZI, 2007), démontre que les actions en faveur de la conservation surviennent souvent grâce à l'appui du public. Avec l'affluence des visiteurs et l'intérêt de la population pour la région du parc marin et les mammifères marins, les opportunités de diffuser l'information scientifique sont multiples, mais exigent l'engagement des chercheurs. À l'avenir, les chercheurs seront appelés à mettre encore plus en priorité les travaux qui permettent de répondre aux enjeux de gestion et de conservation.

\section{NAGER DANS L'INCERTITUDE}

Si la science trouve parfois sa force dans la diffusion, elle comporte par ailleurs des limites. Elle ne peut fournir toutes les réponses, car le milieu marin est intrinsèquement complexe, dynamique, et souvent imprévisible. Les processus clés qui soutiennent les structures et les fonctions des écosystèmes ne sont pas toujours bien compris. Dans ce contexte, l'intégration des résultats scientifiques aux décisions de gestion constitue un 
important défi. Plusieurs décisions doivent être prises sur la base d'informations incomplètes. Là où l'on met le fardeau de la preuve, lorsqu'un enjeu doit être résolu, est effectivement une question centrale du domaine de la conservation du milieu marin (NORSE, 1993). En reconnaissant l'incertitude inhérente aux écosystèmes, il s'avère de plus en plus nécessaire de recourir à une gestion adaptative et au principe de précaution pour encadrer les activités humaines dans un but de conservation du milieu marin.

\section{7. ÉTAT DU PARC MARIN DIX ANS APRÈS SA CRÉATION}

L'état de santé des écosystèmes du parc marin est le résultat d'interactions écologiques multiples, de conditions océaniques variables et de sources de pression nombreuses. Cet état dépend évidemment de l'état du Saint-Laurent et du Saguenay, dont le fonctionnement des écosystèmes a été profondément modifié au cours des derniers siècles. Ainsi, des processus se déroulant à l'échelle locale, régionale ou à celle du bassin versant des Grands Lacs et du Saint-Laurent peuvent avoir un impact déterminant sur l'état du parc marin. Malgré le fait que la gestion d'une aire marine protégée soit limitée à certaines actions à l'intérieur du territoire, c'est par l'éducation, la diffusion des résultats de recherches scientifiques et la gouvernance que l'influence du parc marin peut s'étendre au-delà de ses limites géographiques et administratives.

Une première évaluation de l'état de santé des écosystèmes du parc marin et de l'efficacité des mesures de gestion a été réalisée en 2007 (PMSSL, 2008). Pour ce faire, une approche multidisciplinaire et intégrée s'est imposée, faisant appel à une diversité d'indicateurs provenant de plusieurs domaines. L'analyse a permis de constater que les progrès ont été plus significatifs dans certains domaines, en particulier en ce qui concerne la gouvernance, l'expérience des visiteurs et l'acquisition de connaissances dans divers champs d'études (PMSSL, 2008).

En ce qui concerne l'état des écosystèmes, la gestion du parc marin a permis de ralentir certains processus de dégradation, mais il s'avère complexe de mesurer avec précision ces résultats. Il faut considérer que les écosystèmes subissent les impacts cumulatifs des activités humaines et que les efforts pour réduire ces impacts sont encore récents. Les contaminants sont toujours présents et les activités humaines dans le parc, telles que le trafic maritime et les activités avec prélèvement des ressources biologiques, sont en croissance depuis quelques années. Ensemble, ces sources de pression peuvent avoir des effets cumulatifs et agir en synergie sur les écosystèmes. La stagnation de la population de bélugas démontre que des efforts soutenus sont nécessaires pour engendrer des résultats mesurables et que son rétablissement est un travail de longue haleine.

Quant à l'utilisation écologiquement durable du parc marin, l'implantation de nouveaux outils de gestion pour encadrer les activités sans prélèvement a permis de faire des progrès, notamment pour les activités d'observation en mer. Nous devons cependant maintenir nos efforts afin de susciter l'adhésion de ceux qui pratiquent ces activités. Par ailleurs, l'adaptation de la gestion des activités de prélèvement au concept d'utilisation écologiquement durable et de l'approche écosystémique demeure un grand défi pour la conservation des écosystèmes du parc marin.

\section{CONCLUSION}

Les aires marines protégées sont reconnues dans le monde comme un outil précieux pour favoriser la conservation de la biodiversité des écosystèmes marins, maintenir leur produc tivité, accroître les connaissances et mieux faire apprécier l'importance de ces endroits exceptionnels par le public. Il est aussi largement reconnu que la clé du succès des aires marines protégées passe par la compréhension et l'appui des communautés avoisinantes et des usagers (KELLEHER, 1999).

Les écosystèmes du parc marin fascinent, car ils offrent à la fois un milieu de vie essentiel à plusieurs espèces marines, sont un moteur de l'économie régionale et sont une source d'émerveillement pour les visiteurs et les chercheurs. En somme, l'avenir du parc marin dépend de la continuité de l'engagement des centaines d'intervenants concernés, des communautés côtières ainsi que des milliers de visiteurs qui y viennent chaque année.

Le parc marin a maintenant dix ans, et le temps est venu de faire un bilan des progrès réalisés et des défis qui nous attendent. Il faut reconnaître que beaucoup de chemin a été parcouru depuis son établissement en 1998. Un des grands défis demeure celui de faire en sorte que les connaissances scientifiques servent à prendre des mesures afin de favoriser la conservation de ce milieu exceptionnel qu'est le parc marin. Le numéro spécial de la Revue des Sciences de l'eau et le colloque scientifique "Dix années de science au service de la conservation "nous permettent de faire un grand pas dans la bonne direction. 


\section{REMERCIEMENTS}

Merci à Valérie Busque, Daniel Langlois, Suzan Dionne et Marie-France Richard pour leurs excellents commentaires sur ce texte. Merci aussi à tous les chercheurs qui s'efforcent de rendre leurs connaissances disponibles au public ainsi qu'aux gestionnaires, et qui contribuent à l'atteinte des objectifs du parc marin. J'aimerais souligner en particulier la contribution de notre regretté collègue et ami, le professeur François Saucier, un scientifique reconnu par ses pairs et un expert dans l'art de présenter ses résultats au public. Il nous a fait découvrir les premières images en 3D de la tête du chenal Laurentien, la beauté des "ballerines " à l'embouchure du Saguenay, la force de l'"upwelling" et la musique des clapotis. Finalement, le numéro spécial de la Revue des Sciences de l'eau et le colloque scientifique "Dix années de science au service de la conservation" n'auraient pas été possibles sans le professeur Émilien Pelletier. Depuis près d'une décennie, le professeur Pelletier est engagé au sein du Comité conseil sur la gestion des écosystèmes et la recherche et du Comité de coordination du parc marin. Sa renommée comme chercheur et son influence ont certainement eu un effet positif sur la santé du parc marin!

\section{RÉFÉRENCES}

ARGUS GROUPE CONSEIL (1992). Synthèse et analyse des connaissances relatives aux ressources naturelles $d u$ Saguenay et de l'estuaire du Saint-Laurent-Parc marin du Saguenay, Rapport présenté au Service canadien des parcs, Environnement Canada, région du Québec, pagination multiple.

BEARZI G. (2007). Marine conservation on paper. Conserv. Biol., 21, 1-3.

BOSSÉ L., B. SAINTE-MARIE et J. FOURNIER (1996). Les invertébrés des fonds meubles et la biogéographie du fjord du Saguenay. Rap. Tech. Can. Sci. Halieutiques Aquat., 2132 : vii +45 p.

DIONNE S. (sous la direction de) (2001). Plan de conservation des écosystèmes du parc marin du Saguenay-Saint-Laurent. Parcs Canada, parc marin du Saguenay-Saint-Laurent, 538 p.

DRAINVILLEG.(1968). Lefjord duSaguenay :I. Contribution à l'océanographie. Nat. Can., 95, 809-855.

DUNBAR M.J., D.C. MACLELLAN, A. FILION et D. MOORE (1977). The biogeographic structure of the Gulf of St. Lawrence. Marine Sciences Centre, McGill University,
Montreal. Report prepared for the Marine Studies Section, Parks Canada, 141 p.

EDDS P.L. et J.W. MACFARLANE (1987). Occurrence and general behaviour of balaenopterid cetaceans summering in the St. Lawrence Estuary, Canada. Can. J. Zool., 65, 1363-1376.

FORRESTER W.D. (1974). Internal tides in the St. Lawrence Estuary. J. Mar. Res., 32, 55-66.

INGRAM R.G. (1975). Influence of tidal-induced vertical mixing on the primary productivity in the St. Lawrence Estuary. Mém. Soc. Roy. Sci . Liege 7, 59-74.

KELLEHER G. (1999). Guidelines for marine protected areas. IUCN, Gland, Switzerland and Cambridge, UK, xxiv +107 p.

LAMBERT J.-D. et S. BÉRUBÉ (2002). La pêche sportive hivernale dans le fjord du Saguenay. Rapp. Tech. Can. Sci. Halieutiques Aquat., $2445: \mathrm{x}+58 \mathrm{p}$.

LEBEUF M., B. GOUTEUX, L. MEASURES et S. TROTTIER (2004). Levels and temporal trends (1988-1999) of polybrominated diphenyl ethers in beluga whales (Delphinapterus leucas) from the St. Lawrence Estuary, Canada. Environ. Sci. Technol., 38, 2971-2977.

LYNAS E.M. et J.P. SYLVESTRE (1988). Feeding techniques and foraging strategies of minke whales (Balaenoptera acutorostra) in the St. Lawrence River Estuary. Aquat. Mam., 14, 21-32.

MICHAUD R., V. DE LA CHENELIÈRE, M.-H. D'ARCY et M. MOISAN (2007). Les activités d'observation en mer (AOM) dans l'estuaire du Saint-Laurent: zone de protection marine estuaire du Saint-Laurent et parc marin du Saguenay-Saint-Laurent. Suivi annuel 2006. Rapport final GREMM, Tadoussac, Québec, conjointement avec le parc marin du Saguenay-Saint-Laurent, Québec, et le ministère des Pêches et des Océans du Canada, Québec, Vii + 16 p. + 10 tableaux, 6 figures, 17 cartes et 9 annexes.

MPO (2007). Compte rendu de l'atelier de travail sur le béluga de l'estuaire du Saint-Laurent - revue de programme de suivi des carcasses. Secr. can. consult. sci. MPO, Compte rendu. $2007 / 005$.

NORSE E. A. (éditeur) (1993). Global marine biological diversity: A strategy for building conservation into decision 
making. Center for Marine Conservation, World Conservation Union (IUCN), World Wildlife Fund, United Nations Environment Program, World Bank. Island Press, 383 p.

PELLETIER É, I. DESBIENS, P. SARGIAN, N. CÔTÉ, A. CURTOSI et R. ST-LOUIS (2009). Présence des hydrocarbures aromatiques polycycliques (HAP) dans les compartiments biotiques et abiotiques de la rivière et du fjord du Saguenay. Rev. Sci. Eau, (ce numéro)

PIPPARD L. et H. MALCOLM (1978). Le béluga (Delphinapterus leucas) : observations sur sa distribution, sa population et ses habitats essentiels dans le Saint-Laurent et le Saguenay. Parcs Canada. Contrat 76-190, 161 p. + annexes.

PIPPARD L. (1985). Status of the St. Lawrence River population of beluga, Delphinapterus leucas. Can. FieldNat., 99, 438-450.

PMSSL (2008). Rapport sur l'etat du parc marin $d u$ Saguenay-Saint-Laurent 2007. Parc marin du Saguenay-Saint-Laurent, Tadoussac, Canada, 69 p.

PRESCOTT J. et M. GAUQUELIN (1990). Pour l'avenir du béluga. Dans: Forum international pour l'avenir du béluga. Presses de l'Université du Québec, Sillery, Québec, 345 p.

RAINVILLE L. (1979). Etude comparative de la distribution verticale et de la composition des populations de zooplancton du fjord du Saguenay et de l'estuaire maritime du SaintLaurent. Thèse de maîtrise, Département de biologie, Université Laval, Québec, 175 p.

REID S.J. (1977). Circulation and mixing in the St. Lawrence Estuary near Ille Rouge. Bedford Institute of Oceanography. Rep. Ser. BI-R-77, 36 p.

SAUCIER F.-J., F. ROY, S. SENNEVILLE, G. SMITH, D. LEFAIVRE, B. ZARDJIAN et J.-F. DUMAIS (2009). Modélisation de la circulation dans l'estuaire et le golfe du Saint-Laurent en réponse aux variations du débit d'eau douce et des vents. Rev. Sci. Eau, (ce numéro).

SAVARIA J.-Y., G. CANTIN, L. BOSSÉ, R. BAILEY, L. PROVENCHER et F. PROUST (2003). Compte rendu d'un atelier scientifique sur les mammifères marins, leurs habitats et leurs ressources alimentaires. Mont-Joli (Québec), 3-7 avril 2000, dans le cadre de l'élaboration du projet de zone de protection marine de l'estuaire du
Saint-Laurent. Rapp. Manus. Can. Sci. Halieutiques Aquat. 2647. v + 127 p.

SÉVIGNY J.-M. et C.M. COUILLARD (éditeurs) (1994). Le ford du Saguenay: un milieu exceptionnel de recherche. Rapp. manus. Can. Sci. halieutiques Aquat, $2270: v+118$ p.

SÉVIGNY J.-M., A. VALENTIN, A. TALBOT et N. MÉNARD (2009). Connectivité entre les populations du fjord du Saguenay et celles du golfe du Saint-Laurent. Rev. Sci. Eau, 22, (ce numéro)

SIMARD Y. (2009). Le parc marin du Saguenay-Saint-Laurent : processus océanographique à la base de ce garde-manger unique des baleines du Nord-Ouest Atlantique. Rev. Sci. Eau, 22, (ce numéro)

SIROIS P., G. DIAB, A.-L. FORTIN, S. PLOURDE et N. MÉNARD (2009). Recrutement des poissons dans le fjord du Saguenay. Rev. Sci. Eau, 22, (ce numéro).

THERRIAULT J.-C., L. LEGENDRE et S. DEMERS (1990). Oceanography and ecology of phytoplankton in the St. Lawrence Estuary. Dans El-Sabh, M. I. et N. Silverberg. Oceanography of Large-Scale Estuarine System: The St. Lawrence. Coastal and Estuarine Studies, Springer-Verlag, New York, p. 269-295. 